Annals of Plant and Soil Research 23(3): 341-345 (2021)

https://doi.org/10.47815/apsr.2021.10081

\title{
Wheat genotypes evaluated for GxE interactions in Central Zone of the country by AMMI analysis
}

\author{
AJAY VERMA ${ }^{1}$ AND G.P. SINGH \\ ICAR-Indian Institute of Wheat \& Barley Research, Karnal 132001 Haryana \\ Received: May, 2021: Revised accepted: July, 2021
}

\begin{abstract}
Highly significant effects of environments, GxE interaction and genotypes were observed for cropping years 2017-18 and 2018-19. Further analysis of interactions sum of squares bifurcated into seven significant multiplicative interactions principal components to assess the performances of genotypes as per AMMI based measures. For the first year of study wheat genotypes (G5, G6, G7) had top ranked by EV2, D2, ASV, ASV1 and ASTAB2 measures. MASV \& MASV1 pointed towards G7, G8, G6 wheat genotypes. Association among these measures displayed graphically in a biplot analysis. Largest cluster comprised of D2, D3, D5, D7, ASV, ASV1, ASTAB2, EV2, EV3, EV5, ASTAB3, ASTAB5, ASTAB7 measures. Wheat genotypes (G1, G11, G3) pointed by EV2, D2, ASV, ASV1 and ASTAB2 values for the second year. MASV settled for G11, G7, G13 whereas MASV1 pointed towards G11, G7, G2. Biplot analysis based on first two PC's observed largest group had clubbed measures D2, ASV, ASTAB2, EV5, MASV, MASV1, EV3, D3, D5, D7, EV7, ASTAB3 ASTAB5, ASTAB7. AMMI based measures would be useful to identify and recommend genotypes with high, stable and predictable yield across environments.
\end{abstract}

Key words: AMMI model, ASV, ASTAB, EV, D, MASV, Biplot analysis

\section{INTRODUCTION}

Efficient estimation of main and interaction effects for wheat crop, multi environment trials (MET) had been planned (Bocianowski et al., 2019). An efficient assessment of GxE interaction assisted to determine the stable yield potential of genotypes for specific and general adaptation performance (Mohammadi et al., 2015; Ajay et al., 2019). Statistical procedures have been developed to partition the complex GxE interaction into more meaningful components (Agahi et al., 2020). AMMI model, involves both additive and multiplicative components, separates the additive variance from the multiplicative variance and then applies principal component analysis (PCA) to the interaction portion (Nowosad et al., 2018). AMMI analysis has been effective to capture a large portion of the GxE sum of squares, and present different kinds of opportunities to agricultural researchers (Gauch 2013). Present study was planned with objectives (i) explain AMMI based measures depend on utilization of significant principal components (ii) association analysis among AMMI, yield and adaptability measures.

\section{MATERIALS AND METHODS}

Central Zone comprises of Madhya Pradesh, Chhattisgarh, Gujarat, Rajasthan (Kota

and Udaipur divisions) and Jhansi division of Uttar Pradesh. Nine advanced wheat genotypes twelve locations and thirteen genotypes at fourteen locations were evaluated under field trials during 2017-18 and 2018-19 cropping seasons respectively. Field trials were conducted at research centers in randomized complete block designs with three replications. Recommended agronomic practices were followed to harvest good yield. Details of genotype parentage along with environmental conditions were reflected in Tables 1 \& 2 for ready reference.
EV EVF
SIPC
SIPCF

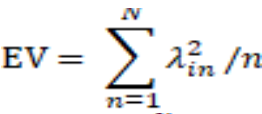
ASV

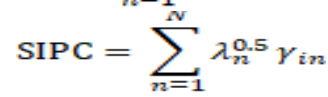
D
ASTAB
ASV1
MASV
MASV1

$$
\begin{aligned}
& \mathrm{ASV}= \\
& {\left[\left(\frac{S S I P C 1}{S S I P C 2} P C I\right)^{2}+(P C 2)^{2}\right]^{1 / 2}} \\
& \mathrm{D}=\sqrt{\sum_{n=1}^{N}\left(\lambda_{n} \gamma_{i n}\right)^{2}}
\end{aligned}
$$$$
A S T A B=\sum_{n=1}^{n} \lambda_{n} Y_{n i}^{2}
$$
$\mathrm{ASV}=$

$$
\left[\frac{S S I P C 1}{S S I P C 2}(P C I)^{2}+(P C 2)^{2}\right]^{1 / 2}
$$

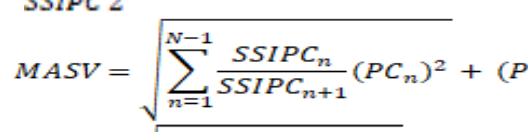$$
M A S V 1=\sqrt{\sum_{n=1}^{N-1}\left(\frac{S S I P C_{n}}{S_{S I P C}+1} P C_{n}\right)^{2}}+\left(P C_{n+1}\right.
$$

${ }^{l}$ Corresponding author: 0184-2209149; 01812267390; ajay.vermal@icar.gov.in 
AMMI analysis was performed using AMMISOFT version 1.0, available at https://scs.cals.cornell. edu/people/hugh-gauch/ and SAS software version 9.3. Analytic measures of adaptability, the relative performance of genetic values (PRVG) and MHVG (Harmonic mean of Genetic Values), relative performance of the genotypic values (MHPRVG) were compared with AMMI based measures (Resende \& Durate, 2007).

$P R V G_{i j}=V G_{i j} / V G_{i}$

$M H V G_{i}=$ Number of environments $/ \sum_{i=1}^{k} \frac{1}{X_{i}}$

$M_{H P R V G}=$ umber of environments $/ \sum_{j=1}^{k} \frac{1}{P R V G_{i j}}$

$V G_{i j}$ was the genotypic value of the i genotype, in the $\mathrm{j}$ environment. Geometric adaptability index (GAI) calculated $a s^{n} \sqrt{\prod_{k=1}^{n} \bar{x}_{k}}$; and genotypes with higher values of GAI were desirable.

\section{RESULTS AND DISCUSSION}

\section{AMMI Analysis}

Highly significant environments, GxE interaction and genotypes effects were observed by ANOVA analysis for the first year of study (Table 3). Interaction effects were further divided into seven significant interaction principal component axes (IPCAs) (Tena et al., 2019). Explained variation of GxE interaction accounted by IPCA's exploited by defined measures, as type-2 measures utilized $64.3 \%$, while type 7 measures accounted for most of variation and utilized to the extent of benefits $98.9 \%$ (Table 3 ). This justifies the use of AMMI derived parameters based on the larger numbers of IPCAs results in the most usage of GxE interaction variations (Nowosad et al., 2016). Large magnitude of GxE interactions for yield found in this investigation are similar to those found in other crops (Mohammadi et al., 2015). The high significance of GE interactions is indicating the studied genotypes exhibited both crossover and non-crossover types of GxE interaction. EV2 pointed towards (G5, G6, G7) as desirable at the same time undesirable genotypes (G3, G4), for values of D2 genotypes were $(\mathrm{G} 5, \mathrm{G} 6, \mathrm{G} 7)$ \& $(\mathrm{G} 3, \mathrm{G} 4)$, whereas as per criterion of SIPC2 were (G9, G7, G1) \& $(G 3, G 4)$ and of ASTAB2 were (G5, G6, G7) \& (G3, G4). Now a days, agronomic concept of stability would be more preferred instead of static concept of stability (Tekdal \& Kendal, 2018). Using first two IPCAs in stability analysis could benefits dynamic concept of stability in identification of the stable high yielder genotypes. ASV and ASV1 recommended (G5, G6, G7) as of stable performance and unsuitable were G3, G4. Considering first two IPCAs in ASV measure used $64.3 \%$ of GxE interaction. The two IPCAs have different values and meanings and the ASV parameter using the Pythagoras theorem and to get estimated values between IPCA1 and IPCA2 scores to produce a balanced measure between the two IPCA scores. Minimum values D7 identified G7, G8, G5 of stable performance while $G 9$ and $G 4$ as undesirable; SIPC7 observed G9, G8, G5 as of stable \& G3, G7 of unstable yield (Tables 5). EV7 pointed towards G7, G8, G5 \& G9, G4. Measure ASTAB7 identified G7, G8, G5 as desirable and G3, G9 for unstable behavior over the studied environments. Composite measure MASV selected G11, G7, G13 as of stable performance and G9, G8 not recommended for cultivation due to unstable yield behavior. Composite measure along with modified version i.e. MASV\& MASV1 pointed towards G7, G8, G6 and G4, G9 for stable and unstable behavior for studied environments respectively.

Second year of study observed diverse nature of environments as $79 \%$ of interaction sum of squares accounted whereas GxE interactions approximated $9 \%$ while genotypes contributed least of total sum of squares. AMMI derived type-2 measures utilized $60.4 \%$, while type-7 accounted for $96.7 \%$ (Table 4). Measures EV2, D2 ASTAB2, ASV and ASV1 pointed towards (G1, G11, G3) as desirable genotypes. Values of D7 identified G1, G2, G7 as of stable performance; SIPC7 favoured G5, G11, G2 (Tables 6). EV7 pointed towards G7, G13, G2 \& ASTAB7 identified G11, G2, G3 as desirable. MASV settled for G11, G7, G13 as of stable performance and Modified version of composite measure MASV1 pointed for G11, G7, G2. PRVG for G5, G8, G1 and MHPRVG for G5, G1, G8 wheat genotypes.

\section{Association analysis by Biplots}

Biplot analysis was performed to define any relationship among the AMMI measures (Shahriari et al., 2018). All measures had distributed among five groups in graphical biplot 
Table 5: AMMI based estimates of genotypes (2017-18)

\begin{tabular}{|c|c|c|c|c|c|c|c|c|c|c|c|c|c|c|c|}
\hline & EV2 & EV7 & D2 & D7 & SIPC2 & SIPC7 & ASTAB2 & ASTAB7 & ASV & ASV1 & MASV & MASV1 & $\mathrm{GAI}$ & PRVG & MHPRVG \\
\hline G1 & 0.0494 & 0.0574 & 4.97 & 7.81 & -1.1798 & 1.2173 & 24.72 & 61.03 & 1.99 & 2.01 & 5.46 & 6.68 & 49.87 & 1.0031 & 0.9983 \\
\hline G2 & 0.0381 & 0.0596 & 4.42 & 6.29 & 0.0673 & 0.4506 & 19.52 & 39.60 & 1.79 & 1.84 & 4.23 & 5.11 & 47.85 & 0.9625 & 0.9575 \\
\hline G3 & 0.1515 & 0.0624 & 8.98 & 9.74 & 3.8755 & 4.3541 & 80.70 & 94.78 & 3.71 & 3.90 & 4.63 & 5.06 & 51.90 & 1.0472 & 1.0360 \\
\hline G4 & 0.1214 & 0.0625 & 7.77 & 8.55 & 2.4334 & 1.8232 & 60.35 & 73.12 & 3.10 & 3.11 & 6.11 & 7.99 & 49.31 & 0.9930 & 0.9856 \\
\hline G5 & 0.0034 & 0.0515 & 1.34 & 5.27 & -0.1727 & -2.0001 & 1.80 & 27.82 & 0.55 & 0.57 & 4.09 & 4.85 & 48.96 & 0.9835 & 0.9811 \\
\hline G6 & 0.0089 & 0.0611 & 2.18 & 5.84 & -0.9629 & 1.0579 & 4.74 & 34.08 & 0.90 & 0.94 & 4.02 & 4.68 & 51.69 & 1.0387 & 1.0356 \\
\hline G7 & 0.0112 & 0.0352 & 2.42 & 4.30 & -1.2808 & 2.0776 & 5.87 & 18.50 & 1.00 & 1.04 & 2.90 & 3.37 & 51.35 & 1.0311 & 1.0295 \\
\hline G8 & 0.0235 & 0.0468 & 3.53 & 5.27 & 1.0226 & -2.1305 & 12.46 & 27.76 & 1.46 & 1.53 & 3.49 & 4.06 & 48.09 & 0.9659 & 0.9640 \\
\hline G9 & 0.0927 & 0.0635 & 6.86 & 8.70 & -3.8027 & -6.8502 & 47.07 & 75.73 & 2.77 & 2.82 & 5.76 & 7.13 & 48.43 & 0.9749 & 0.9687 \\
\hline
\end{tabular}

Table 6: AMMI based estimates of genotypes (2018-19)

\begin{tabular}{|c|c|c|c|c|c|c|c|c|c|c|c|c|c|c|c|}
\hline & EV2 & EV7 & D2 & D7 & SIPC2 & SIPC7 & ASTAB2 & ASTAB7 & ASV & ASV1 & MASV & MASV1 & GAI & PRVG & MHPRVG \\
\hline G1 & 0.0004 & 0.0480 & 2.05 & 18.87 & 0.0180 & -0.2637 & 0.49 & 60.84 & 0.2981 & 0.3757 & 4.9605 & 5.5069 & 57.99 & 1.0266 & 1.0222 \\
\hline G2 & 0.0071 & 0.0266 & 7.59 & 15.49 & -0.0494 & -0.5309 & 7.20 & 31.23 & 1.0239 & 1.1340 & 3.8996 & 4.5609 & 56.06 & 0.9914 & 0.9892 \\
\hline G3 & 0.0071 & 0.0512 & 7.34 & 18.91 & 0.1518 & 1.0665 & 6.84 & 36.29 & 0.9714 & 1.0306 & 5.0786 & 5.5819 & 57.30 & 1.0149 & 1.0093 \\
\hline G4 & 0.0432 & 0.0344 & 19.07 & 24.35 & -0.4067 & -0.1017 & 44.93 & 77.88 & 2.6021 & 2.9518 & 5.1865 & 5.9355 & 56.76 & 1.0065 & 0.9988 \\
\hline G5 & 0.0808 & 0.0321 & 29.77 & 31.21 & -0.5171 & -0.9612 & 102.81 & 115.85 & 4.3105 & 5.4146 & 5.1725 & 7.1945 & 58.71 & 1.0420 & 1.0323 \\
\hline G6 & 0.0334 & 0.0364 & 15.66 & 22.18 & 0.1922 & 0.3036 & 31.47 & 69.43 & 2.0507 & 2.1147 & 5.1391 & 5.6567 & 56.71 & 1.0046 & 0.9989 \\
\hline G7 & 0.0174 & 0.0169 & 14.03 & 18.03 & 0.2232 & 0.1968 & 22.68 & 39.37 & 2.0416 & 2.5841 & 3.3107 & 4.1327 & 56.24 & 0.9953 & 0.9918 \\
\hline G8 & 0.0634 & 0.0509 & 23.17 & 29.29 & -0.0976 & -0.1091 & 66.21 & 110.78 & 3.1670 & 3.6055 & 6.1758 & 7.1726 & 57.98 & 1.0295 & 1.0185 \\
\hline G9 & 0.1060 & 0.0540 & 28.45 & 33.12 & 0.5910 & -0.0084 & 102.67 & 143.16 & 3.7724 & 4.0175 & 7.0454 & 7.7519 & 56.96 & 1.0152 & 0.9962 \\
\hline G10 & 0.0204 & 0.0369 & 13.93 & 22.24 & 0.0134 & 0.0967 & 23.24 & 67.27 & 1.9590 & 2.3505 & 5.1830 & 6.3819 & 57.08 & 1.0120 & 1.0047 \\
\hline G11 & 0.0031 & 0.0371 & 5.00 & 12.93 & -0.1065 & -0.6020 & 3.12 & 9.13 & 0.6756 & 0.7512 & 2.9294 & 3.1976 & 56.78 & 1.0041 & 1.0019 \\
\hline G12 & 0.0940 & 0.0518 & 31.75 & 33.88 & -0.2115 & 0.1874 & 117.49 & 127.95 & 4.5779 & 5.7155 & 5.7379 & 7.6360 & 49.68 & 0.8834 & 0.8718 \\
\hline G13 & 0.0238 & 0.0237 & 16.50 & 19.88 & 0.1991 & 0.7261 & 31.30 & 50.84 & 2.4090 & 3.0612 & 3.8635 & 5.0370 & 54.98 & 0.9745 & 0.9677 \\
\hline
\end{tabular}




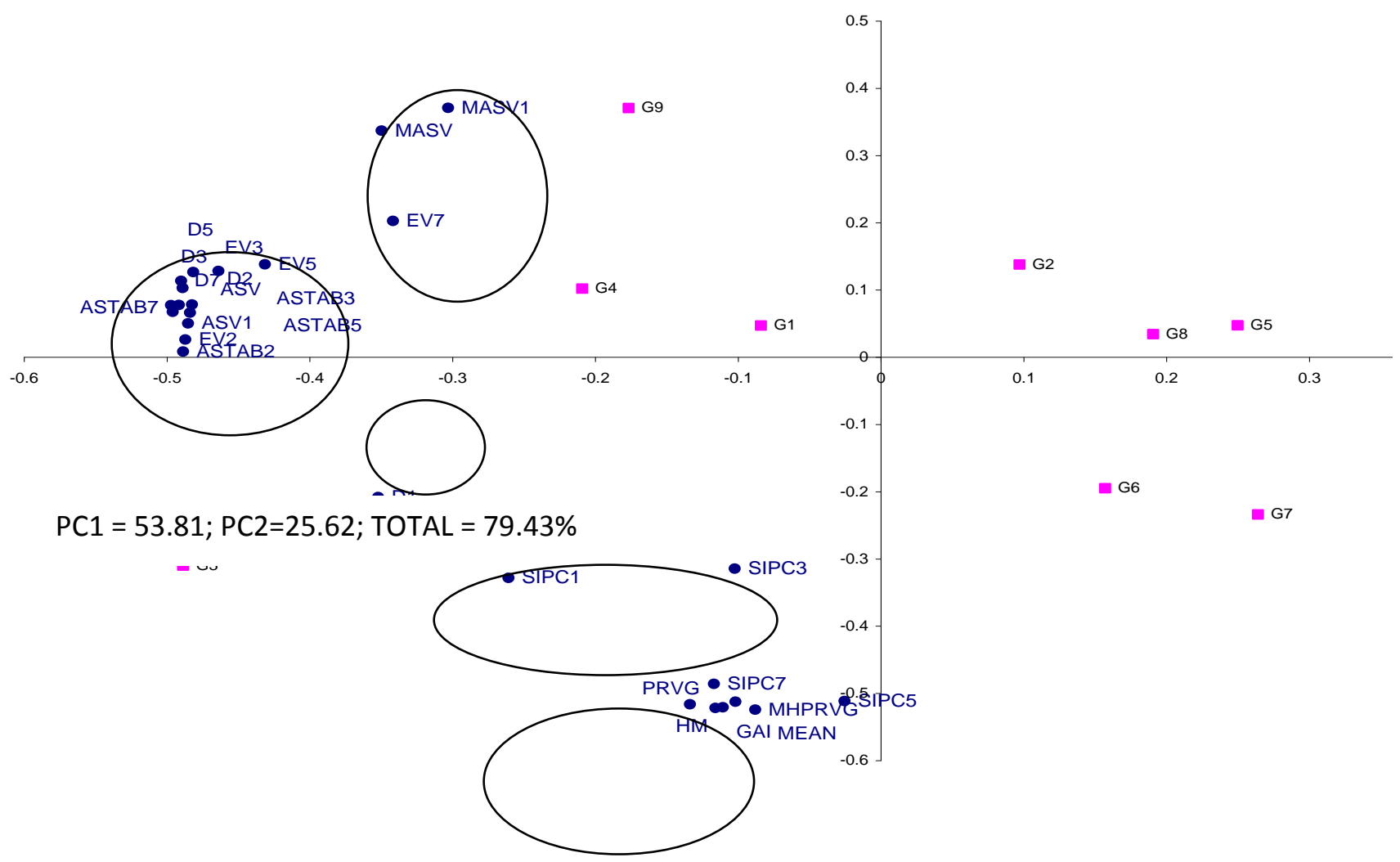

Figure 1: Biplot analysis of genotypes and AMMI based estimates (2017-18)

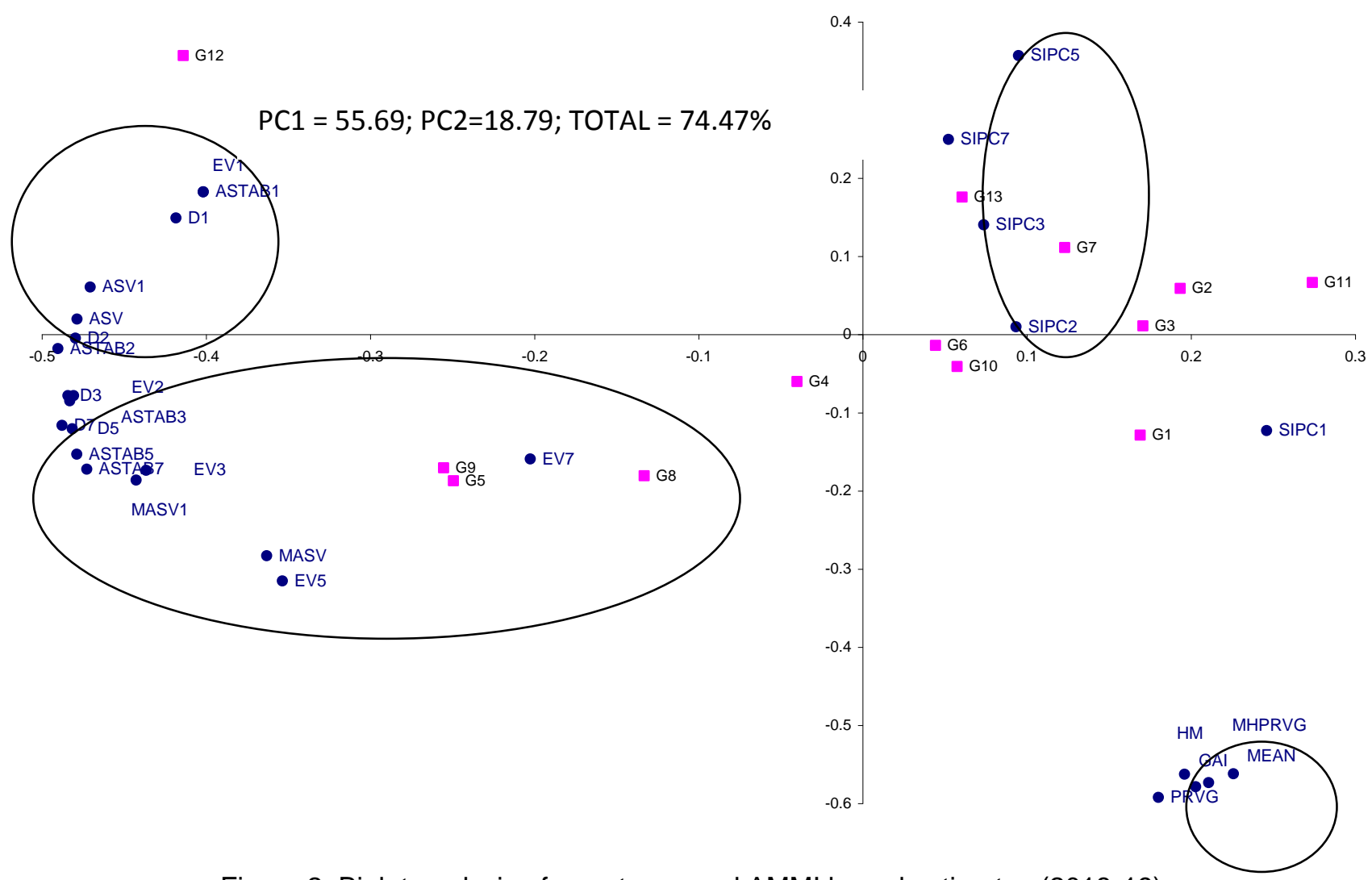

Figure 2: Biplot analysis of genotypes and AMMI based estimates (2018-19) 
analysis. The relationship among these estimates is graphically displayed in a plot of PC1 versus PC2 as these components accounted for more than $79 \%$ of total variations sum of squares among the estimates (Figure 1). Largest group consisted of measures as D2, D3, D5, D7,ASV, ASV1, ASTAB2,EV2, EV3, EV5, ASTAB3, ASTAB5, ASTAB7.

Biplot analysis for second year observed that AMMI based measures had distributed among four quadrants in as graphical representations based on first two significant principal components accounted for more than $74 \%$ of variations among the estimates. Measures could be divided into four major clusters (Figure 2). Largest group consisted of clubbed 16 measures as D2, ASV, ASTAB2, EV5, MASV, MASV1, EV3, D3, D5, D7, EV7,

\section{REFERENCES}

Agahi K., Jafar Ahmadi, Hassan Amiri Oghan, Mohammad Hossein Fotokian and SedighehFabriki Orang (2020) Analysis of genotype $\times$ environment interaction for seed yield in spring oilseed rape using the AMMI model. Crop Breeding and Applied Biotechnology 20(1): e26502012

Ajay B. C., J. Aravind, R. Abdul Fiyaz, Narendra Kumar, Chuni Lal, K. Gangadhar, Praveen Kona, M. C. Dagla and Bera S. K. (2019) Rectification of modified AMMI stability value (MASV) Indian Journal. Genetics. 79(4): 726-731

Bocianowski, J., Niemann, J., Nowosad, K. (2019) Genotype-by-environment interaction for seed quality traits in interspecific cross-derived Brassica lines using additive main effects and multiplicative interaction model. Euphytica 215:7.

Gauch HG (2013). A Simple Protocol for AMMI Analysis of Yield Trials. Crop Science 53:1860-1869.

Mohammadi, M., Sharifi,P., Karimizadeh,R., Jafarby, J.A., Khanzadeh, H., Hosseinpour, T., Poursiabidi, M.M., Roustaii, M., HassanpourHosni, M., Mohammadi, P. (2015) Stability of grain yield of durum wheat genotypes by AMMI model. Agriculture \& Forestry 61(3): 181-193.

Nowosad, K., Liersch, A., Popławska, W., Bocianowski, J.(2016) Genotype by
ASTAB3 ASTAB5, ASTAB7. GxE interaction analysis by $A M M I$ provided a better understanding genotypes performance and discriminate environments as per the adaptability to specific environments. AMMI based measures relates to a concept of yield and stability of wheat genotypes. These measures would be useful to wheat researchers attempt to identify and recommend genotypes with high, stable and predictable yield across environments.

\section{ACKNOWLEDGEMENT}

The wheat genotypes were evaluated at coordinated centers of AICW\&BIP across the country. Authors sincerely acknowledge the hard work of all the staff for field evaluation and data recording.

environment interaction for seed yield in rapeseed (Brassica napus L.) using additive main effects and multiplicative interaction model. Euphytica 208:187-194

Nowosad, K., Tratwal, A., Bocianowski, J. (2018) Genotype by environment interaction for grain yield in spring barley using additive main effects and multiplicative interaction model. Cereal Research Communications 46(4):729-738.

Resende MDV de and Duarte JB. (2007) Precision and quality control in variety trials. Pesquisa Agropecuaria Tropical. 37(3): 182-194.

Shahriari Z, Heidari B, Dadkhodaie A. (2018). Dissection of genotype $\times$ environment interactions for mucilage and seed yield in Plantago species: Application of AMMI and GGE biplot analyses. PLoS ONE 13(5): e0196095

Tekdal, S., Kendal, E. (2018) AMMI Model to Assess Durum Wheat Genotypes in MultiEnvironment Trials, Journal of Agricultural Science and Technology 20: 153-166.

Tena, E., Goshu, F., Mohamad, H., Tesfa, M., Tesfaye, D., Seife, A., (2019). Genotypexenvironment interaction by AMMI and GGE-biplot analysis for sugar yield in three crop cycles of sugarcane (Saccharumofficinirum L.) clones in Ethiopia. Cogent Food \& Agriculture 5: 114. 